\title{
Hydroxychloroquine immediate release tablets: Formulation and evaluation of a solid dosage form
}

Tomaž Einfalt ${ }^{1}$, Pascal Detampel ${ }^{1}$, Daniel Häussinger ${ }^{2}$, Jens Casper ${ }^{1}$, Christoph Meier ${ }^{3}$, Maxim Puchkov $^{1}$, Jörg Huwyler ${ }^{1}$

${ }^{1}$ Department of Pharmaceutical Technology, University of Basel, Klingelbergstrasse. 50, 4056 Basel, Switzerland;

${ }^{2}$ Department of Chemistry, University of Basel, St. Johanns-Ring 19, 4056 Basel, Switzerland;

${ }^{3}$ Department of Clinical Pharmacy, University of Basel, Klingelbergstrasse. 50, 4056 Basel, Switzerland;

*Correspondence:

Prof. Dr. Jörg Huwyler

University of Basel

Division of Pharmaceutical Technology

Klingelbergstrasse 50, 4056 Basel, Switzerland

Tel: +41 6120715 13; E-mail: joerg.huwyler@unibas.ch 


\section{Abstract}

Hydroxychloroquine (HCQ) is a quinoline derivate used for the treatment of malaria and rheumatoid disorders. During early phases of the SARS-CoV2 (COVID-19) pandemic, preliminary and later not substantiated reports suggested that HCQ might benefit COVID-19 patients. This had sparked a worldwide and rapidly rising demand for HCQ drug products. Consequently, patients with pre-existing rheumatic diseases in Switzerland were confronted with an acute drug shortage.

We have therefore designed, produced and characterized a generic HCQ drug formulation. The proposed HCQ formulation can be manufactured by using a minimal number of operation steps (mixing, wet granulation, sieving, blending, compression) and readily available pharmaceutical excipients.

HCQ tablets were manufactured by granulation of the active pharmaceutical ingredient (API), blending with the external phase and compaction using a non instrumented single punch tablet press. Analytics and identification of the API was performed by a combination of NMR, ESI-MS, FTIR and HPLC. HCQ tablets met the quality criteria for an immediate release HCQ dosage form.

We hope that free access to non-proprietary protocols covering analytical procedures, formulation design, and manufacturing instructions for HCQ tablets will help to bridge existing and future supply chain gaps.

Keywords: hydroxychloroquine, tablet, immediate release, solid dosage form 


\section{Abbreviations}

API, Active Pharmaceutical Ingredient; BCS, Biopharmaceutics Classification System; COVID19, Coronavirus disease; COSY, Correlated Spectroscopy; EDQM, European Directorate for the Quality of Medicines; ESI-MS, Electrospray Ionization Mass Spectroscopy; Eur. Pharm., European Pharmacopoeia; FDA, Food and Drug Administration; FTIR, Fourier-Transform Infrared Spectroscopy; HPLC, High Performance Liquid Chromatography; HP, Hydroxypropylcellulose; HCQ, Hydroxychloroquine; HSQC, Heteronuclear Single Quantum Coherence; NOSY, Nuclear Overhauser Effect Spectroscopy; NMR, Nuclear Magnetic Resonance; UPLC, Ultra Performance Liquid Chromatography; USP, United States Pharmacopoeia; UV-Vis, Ultraviolet-Visible spectroscopy. 


\section{Introduction}

Hydroxychloroquine (HCQ) and chloroquine are clinically used as antimalarial drugs $(1,2)$ and to treat rheumatoid arthritis, systemic lupus erythematosus, and other inflammatory rheumatic diseases (3) . HCQ was first synthesized in 1950 and made available as a drug in 1955. Hydroxychloroquine sulfate used in the present study is classified as a BCS-3 drug, with high solubility (about $200 \mathrm{mg} / \mathrm{mL}$ in water) and absorption in the upper $\mathrm{Gl}$ tract. The oral bioavailability is in the range of $75 \%$ (4). Due to accumulation in the lysosomal compartment (1), HCQ has a high volume of distribution of approximately $30 \mathrm{~L} / \mathrm{kg}(5)$ leading to a long halflife in the circulation ( 30 days). Indeed, it has been estimated that it takes 4 month of daily dosing to achieve $90 \%$ of steady-state blood concentrations (4). In this study, single oral administration of HCQ sulfate $(200 \mathrm{mg})$ led to mean maximum plasma concentrations of $244 \mathrm{ng} / \mathrm{mL}$ within 2 to 4.5 hours. This corresponds to $25 \%$ of steady-state blood concentrations after chronic administration. Side effects associated with HCQ administration include gastrointestinal and abdominal discomfort. In rare cases, drug induced cardiotoxicity, QT prolongation and myopathy were observed (6).

HCQ gained global attention as a potential treatment of COVID-19 infections (7) based on promising but preliminary reports. Unfortunately, subsequent multicenter randomized trials found no differences in outcomes as compared to placebo (8). The initial hype nevertheless caused a rapidly increasing demand. In Switzerland, stocks of Plaquenil ${ }^{\circledR}$ and the co-marketing drug Zentiva ${ }^{\circledR}$ (i.e. coated tablets containing $200 \mathrm{mg}$ of $\mathrm{HCQ}$ ) were sold out and patients suffering from pre-existing chronic diseases were confronted with an acute drug shortage. While tablets (i.e. the commonly prescribed oral dosage form) were scarce, the active pharmaceutical ingredient HCQ sulfate was still available in Switzerland and could be obtained in sufficient quantity.

In view of current and potential future HCQ drug shortages worldwide, we therefore decided to design a generic tablet formulation of HCQ. It was our aim to define a simple and robust process, which should rely on minimal unit of operation manufacturing steps and readily available excipients. Considering product-specific FDA guidances, hydroxychloroquine sulfate based formulations can request bioequivalence waivers for immediate release formulations 
(9). Therefore, our formulation is designed to be a generic alternative to the FDA approved Plaquenil ${ }^{\circledR}$. In response to the COVID-19 crisis, non-proprietary analytical and manufacturing protocols are hereby provided.

\section{Materials and Methods}

\section{Materials}

Hydroxychloroquine sulphate (HCQ) was purchased from SCl Pharmtech (Taoyuan City, Taiwan). Calcium hydrogen phosphate, anhydrous (Emcompress) was donated by Glatt AG (Binzen, Germany). Hydroxypropyl cellulose low viscosity (Disso HPC-L, Nippon Soda Co. Ltd, Tokyo, Japan) was received as a gift from Glatt AG (Binzen, Germany). Crosscarmellose sodium NF (Ac-Di-Sol SD-711 NF) was purchased from FMC International (Philadelphia, PA). Magnesium stearate was purchased from Haensler AG (Herisau, Switzerland). Colloidal silica (Aerosil Pharma 200 VV) was purchased from Evonik Industries (Essen, Germany).

\section{Analytical methods}

NMR spectra were recorded on a Bruker Avance III spectrometer (Billerica, MA) operating at 600.13 $\mathrm{MHz}$ proton frequency, equipped with a direct observe BBFO smart probe with selfshielded z-gradient. $\mathrm{HCQ}$ samples were dissolved in $\mathrm{D}_{2} \mathrm{O}$ with internal referencing to TSP-d4 $\left(\delta{ }^{1} \mathrm{H}:-0.083 \mathrm{ppm}, \delta^{13} \mathrm{C}:-2.74 \mathrm{ppm}(10)\right.$; the final tablet was finely powdered using an agate mortar and pestle, $17.63 \mathrm{mg}$ of the resulting powder were suspended in $1.0 \mathrm{~mL}$ of $\mathrm{D}_{2} \mathrm{O}$, homogenized on a vortex mixer, centrifuged at 13'000 rpm for $5 \mathrm{~min}$ and the supernatant was carefully collected for NMR and ESI-MS analyses. HPC samples were dissolved in acetoned6 using residual solvent signals as references $\left(\delta^{1} \mathrm{H}: 2.05 \mathrm{ppm}, \delta^{13} \mathrm{C}: 29.92 \mathrm{ppm}\right)$. Spectra were recorded at $298 \mathrm{~K}$ and the temperature was calibrated using a methanol standard showing accuracy within $+/-0.2 \mathrm{~K}$. The chemical structure of $\mathrm{HCQ}$, numbering of carbon atoms, and a summary of NMR chemical shifts are provided in Figure 1.

Infrared (IR) spectra were recorded on a Shimadzu FTIR Tracer 100 spectrometer (Shimadzu, Kyoto, Japan) with a diamond anvil. IR spectra were semi-automatically baseline corrected using a multipoint algorithm. Absorption bands are given in wave numbers $v \sim\left[\mathrm{cm}^{-1}\right]$. 
UV-VIS spectrum was recorded with UPLC Shimadzu Nexera, SPD-M30A PDA model. Start wavelength was set to $190 \mathrm{~nm}$, end wavelength was set to $700 \mathrm{~nm}$, slit width was $8 \mathrm{~nm}$.

ESI-MS spectra were recorded on a Bruker maXis 4G QTOF ESI mass spectrometer. Spectra were recorded in the positive ion mode using pure API or a finely powdered tablet.

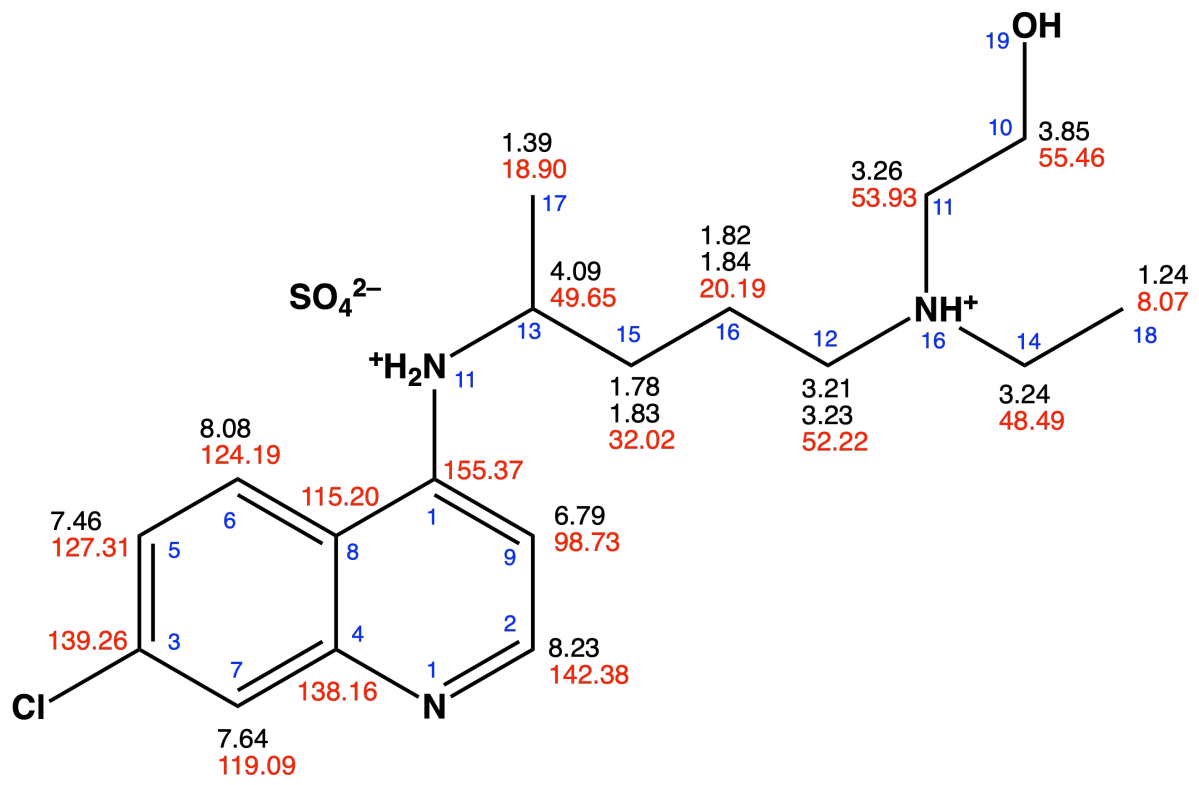

Figure 1. Chemical structure, numbering of carbon atoms, and NMR chemical shifts of hydroxychloroquine (HCQ). Hydroxychloroquine sulfate $\left(\mathrm{C}_{18} \mathrm{H}_{26} \mathrm{ClN}_{3} \mathrm{O} \cdot \mathrm{H}_{2} \mathrm{SO}_{4}\right)$ used in the present study has a MW of $433.95 \mathrm{~g} / \mathrm{mol} .{ }^{1} \mathrm{H}-\mathrm{NMR}$ spectra were recorded in $\mathrm{D}_{2} \mathrm{O}$ at $298 \mathrm{~K}$ and $600.1 \mathrm{MHz}$ (black numbers). ${ }^{13} \mathrm{C}\left\{{ }^{1} \mathrm{H}\right\}-N M R$ spectra were recorded in $\mathrm{D}_{2} \mathrm{O}$ at $298 \mathrm{~K}$ and 150.9 $\mathrm{MHz}$ (red numbers).

Preparation of tablets

HCQ, calcium hydrogen phosphate, and HPC-L were weighed and pre-mixed in a Mycromix high shear mixer (Oystar Hüttlin, Schopfheim, Germany) at a mixer and chopper speed of 116 rpm and $1420 \mathrm{rpm}$, respectively, for $5 \mathrm{~min}$. Subsequently, the powder was wet granulated by adding deionized water to the granulation vessel at mixer and chopper speed up to $160 \mathrm{rpm}$ and $2000 \mathrm{rpm}$, respectively, for $28 \mathrm{~min}$. The obtained granulate of $\mathrm{HCQ}$ were dried at $80^{\circ} \mathrm{C}$ for 25 min and milled with a screen mill (Fitz mill model L1A, Fitz Patrick, Waterloo, Canada). Sieved $(1.25 \mathrm{~mm})$ and milled granules were blended together. The external phase of crosscarmellose sodium, colloidal silica, and finally magnesium stearate was added by 
subsequent mixing of each component in a Turbula powder blender T2C (W.A. Bachofen, Muttenz, Switzerland) for 5 minutes each. Complete formulation of the developed HCQ tablets is shown in Table 1.

Table 1. Composition of the HCQ tablets.

\begin{tabular}{lccc}
\hline Component & \multicolumn{2}{c}{ Amount per tablet } & Amount per batch \\
& $\mathrm{mg}$ & $\%, \mathrm{w} / \mathrm{w}$ & $\mathrm{g}$ \\
\hline Hydroxychloroquine sulphate & 200 & 68.97 & 1000 \\
Calcium hydrogen phosphate, & 62 & 21.38 & 310 \\
dihydrate & & & \\
HPC-L & 11 & 3.79 & 55 \\
Crosscarmellose sodium & 13 & 4.48 & 65 \\
Magnesium stearate & 3 & 1.03 & 15 \\
Colloidal silica & 1 & 0.34 & 5 \\
& & & \\
Tablet weight & 290 & 100 & 1450 \\
Total batch size & & &
\end{tabular}

The resulting granulate was compressed using a single punch press (Korsch EKO, Berlin, Germany) with $9 \mathrm{~mm}$ round tooling, with curvature radius of $9 \mathrm{~mm}$ (Figure 4). The compression speed was set to $30 \mathrm{rpm}$. Tablet target weight was set to $290 \mathrm{mg}$ with target hardness of $70 \mathrm{~N}$ (Dr. Schleuniger Tablet Tester 8 M, Pharmatron AG, Thun, Switzerland). Obtained tablets had a height of $4.49 \mathrm{~mm}$.

\section{Characterization of tablets}

HCQ tablets size parameters were determined using a digital scale caliper (Matrix Handels, Germany). Individual mass ( $n=10$ ) of HCQ tablets was measured on an electronic balance (AX 204 Delta Range, Mettler Toledo, Switzerland).

\section{Uniformity of mass}

HCQ tablets weight and mass uniformity were determined according to the method provided by Eur. Pharm. 9.0, 2.9.40. In brief, 20 tablets were individually weighted on an electronic balance (KERN Balance ABT 220-5DNM; Kern \& Sohn, Balingen, Germany). Weight of all of the selected tablets had a lower than $5 \%$ deviation from average. 


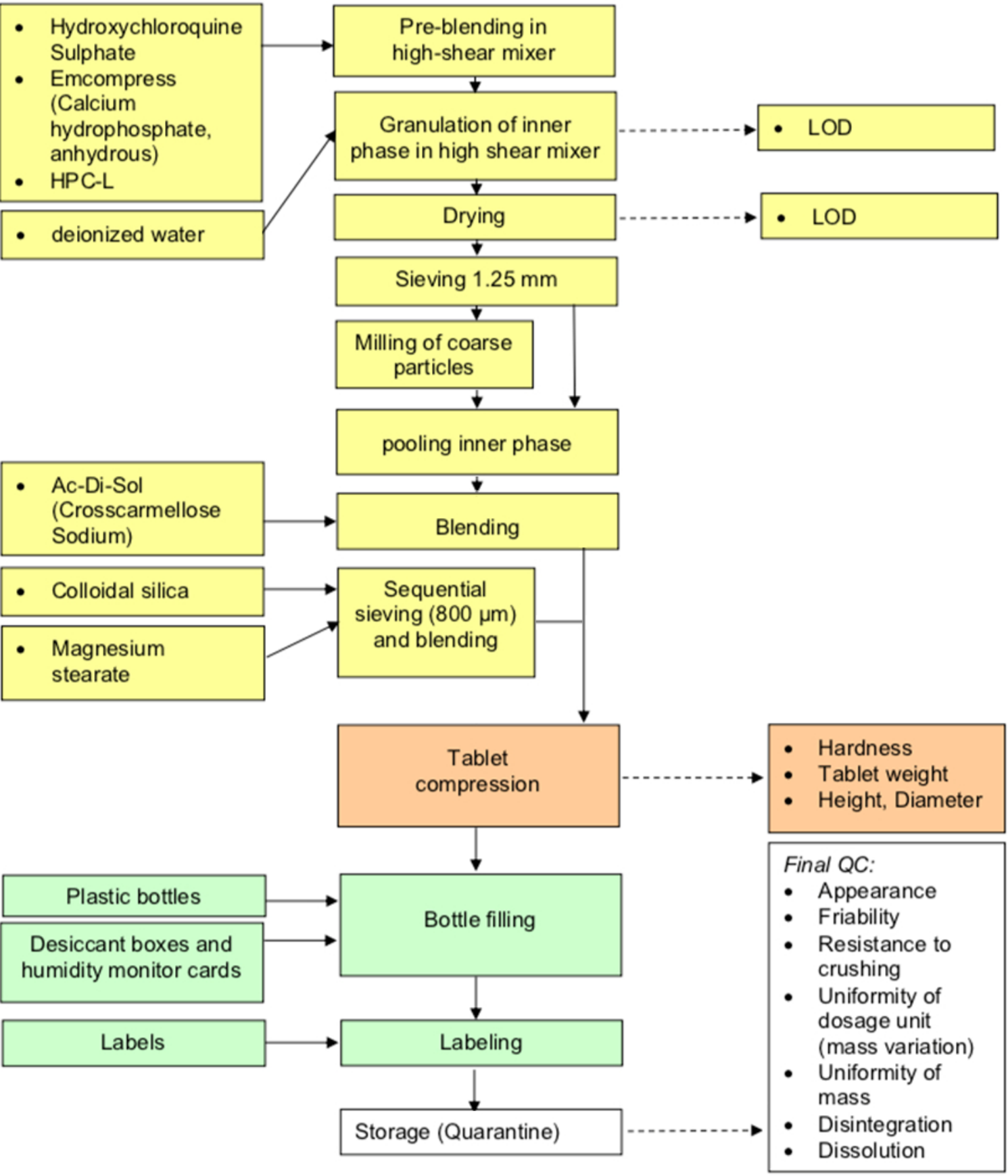

Figure 2. Production flowchart of the HCQ tablets.

\section{Drug content and uniformity of dosage forms}

Drug content of HCQ tablets $(n=30)$ was evaluated by the HPLC-UV method proposed in USP29. Briefly, ten HCQ tablets were randomly selected from the sample of 30 tablets, independently dissolved in $200 \mathrm{~mL}$ of a methanol and water mixture (1:1). Dissolved solutions 
were filtered (0.22 $\mu \mathrm{m}$ syringe filters) and further diluted 1:20 using HPLC mobile phase. For chromatographic separation by HPLC (UPLC Shimadzu Nexera 2), a C18 column was used (Waters Symetry C18, $5 \mu \mathrm{m}, 4.6 \times 100 \mathrm{~mm}$; Waters, Milford, USA). The column temperature was set to $25^{\circ} \mathrm{C}$. Mobile phase was prepared according to USP29 (methanol : acetonitrile : water : phosphoric acid $=100: 100: 800: 2$ ). 96 mg of sodium-1-pentanesulfate was added to $1 \mathrm{~L}$ of the resulting solution and filtered. Drug concentrations were determined based on chromatographic peak areas according to USP29. A calibration curve was prepared by dissolving and diluting the USP analytical standard of HCQ in methanol and water (1:1 v/v). Tablet samples were prepared by dissolving each tablet in $200 \mathrm{~mL}$ of methanol water using an ultrasonic bath. The resulting solution was further diluted into mobile phase at a concentration of $0.05 \mathrm{mg} / \mathrm{mL}$ for injection.

\section{Disintegration of tablets}

The disintegration test was carried out using a Sotax DT2 apparatus (Sotax, Aesch, Switzerland). One HCQ tablet was introduced in each of the 6 baskets with a disc to prevent flotation (European Directorate for the Quality of Medicines, EDQM, 2012). Medium was distilled water.

\section{Friability}

Friability was tested using a TA200 apparatus (Erweka, Langen, Germany) using uncoated HCQ tablets subjected to $25 \mathrm{rpm}$ and 100 revolutions according to EDQM, 2012. Friability was calculated for HCQ tablets according to Eq. (2):

$$
F=\frac{W_{i}-W_{f}}{W_{i}}
$$

Where $W_{i}$ is the initial weight $(\mathrm{mg})$ and $W_{f}$ is the final weight $(\mathrm{mg})$ after friability testing has been completed. 


\section{Dissolution studies}

Drug release from HCQ tablets was measured using an USP II apparatus (Sotax AT7) at $37.0 \pm$ $0.5^{\circ} \mathrm{C}$. Dissolution conditions were according to USP 29. The dissolution buffers was distilled water. Samples were taken at one minute time intervals with a Sotax piston pump CY7 and were analyzed by UV-Vis spectrophotometry (Ultrospec 3100; Amersham Biosciences, Buckinghamshire, UK). The spectrophotometer was equipped with $1 \mathrm{~mm}$ quartz flow-through cuvettes.

\section{Results and discussion}

\section{Results of identity tests}

Hydroxychloroquine sulfate ( $\mathrm{HCQ}$, Figure 1) was acquired from an API manufacturer. A certificate of analysis was provided in compliance with USP/EP specifications. HCQ is a white or slightly yellowish crystalline powder. It is freely soluble in water and practically insoluble in ethanol (96\%) and methylene chloride. According to USP 29 and EP 10, HCQ can be identified by Fourier Transform Infrared absorption spectroscopy (FTIR) using the neat solid on a diamond anvil (Figure 3). Characteristic peaks are given at 3202, 3088, 2970, 1612, 1444, $1365,1342,1248,1215,1082,1024,1006,823,604$ and $589 \mathrm{~cm}^{-1}$ (Figure 1). In addition to FTIR, the UV-HPLC assay described in USP 29 can be applied in to verify HCQ identity. The spectrophotometric scan of HCQ sulfate should show characteristic absorption maxima at 220 $\mathrm{nm}, 234 \mathrm{~nm}, 330 \mathrm{~nm}$ and $342 \mathrm{~nm}$. HCQ should elute as a single peak. Whenever possible, nuclear magnetic resonance (NMR) spectroscopy and high-resolution mass spectrometry should be applied in addition to FTIR and UV-HPLC to confirm the molecular API identity. This method can be applied even when reference substances/spectra are not available. In the present study, NMR served as a mean to determine both purity and drug content.

Identity of HCQ was unambiguously determined by routine one- and two-dimensional NMR spectroscopy $\left({ }^{1} \mathrm{H},{ }^{13} \mathrm{C}\left\{{ }^{1} \mathrm{H}\right\}\right.$, COSY, NOESY, HSQC, HMBC). The NMR spectra of HCQ sulfate (Figure 1) were assigned as follows: ${ }^{1} \mathrm{H}-\mathrm{NMR}\left(\mathrm{D}_{2} \mathrm{O}, 298 \mathrm{~K}, 600.1 \mathrm{MHz}\right): \delta=1.24\left(\mathrm{t},{ }^{3} \mathrm{~J}=7.3 \mathrm{~Hz}, 3 \mathrm{H}\right.$, H21), 1.39 ( $d, 3 \mathrm{~J}=5.2 \mathrm{~Hz}, 3 \mathrm{H}, \mathrm{H} 22$ ), 1.78/1.83 (m, 2H, H13a, H13b), 1.82/1.84 (m, 2H, H14a, $\mathrm{H} 14 b), 3.21 / 3.23$ (m, 2H, H15a, H15b), 3.24 (m, 2H, H2O), 3.26 (m, 2H, H17), 3.85 (t, ${ }^{3} \mathrm{~J}=5.2 \mathrm{~Hz}$, 
$2 \mathrm{H}, \mathrm{H} 18), 4.09$ (m, 1H, H12), 6.79 (d, $\left.1 \mathrm{H},{ }^{3} \mathrm{~J}=7.4 \mathrm{~Hz}, \mathrm{H3}\right), 7.46$ (dd, $1 \mathrm{H},{ }^{3} \mathrm{~J}=9.1 \mathrm{~Hz},{ }^{4} \mathrm{~J}=2.1 \mathrm{~Hz}, \mathrm{H6}$ ), $7.64\left(\mathrm{~d}, 1 \mathrm{H},{ }^{2} \mathrm{~J}=2.1 \mathrm{~Hz}, \mathrm{H} 8\right), 8.08\left(\mathrm{~d}, 1 \mathrm{H},{ }^{3} \mathrm{~J}=9.1 \mathrm{~Hz}, \mathrm{H} 5\right), 8.23\left(\mathrm{~d}, 1 \mathrm{H},{ }^{3} \mathrm{~J}=7.4 \mathrm{~Hz}, \mathrm{H} 2\right) ;{ }^{13} \mathrm{C}\left\{{ }^{1} \mathrm{H}\right\}-N M R$ $\left(\mathrm{D}_{2} \mathrm{O}, 298 \mathrm{~K}, 150.9 \mathrm{MHz}\right): \delta=8.07$ (C21), 18.90 (C22), 20.19 (C14), 32.02 (C13), 48.49 (C20), 49.65 (C12), 52.22 (C15), 53.93 (C17), 55.46 (C18), 98.73 (C3), 115.20 (C10), 119.09 (C8), 124.19 (C5), 127.31 (C6), 138.16 (C9), 139.26 (C7), 142.38 (C2), 155.37 (C4). (Figure 2, Supplementary Figure 1, 2)

Identical NMR spectra of the pure API and the final tablet do not only demonstrate unambiguously the identity of the API, but also its stability during the entire formulation process. Purity of the API was $>99 \%$ as determined by liquid chromatography in agreement with the manufacturer's certificate of analysis (Supplementary Figure 3).

NMR is also a valid method to confirm the absence of impurities listed in the EP for HCQ. The EP lists the following chemical impurities related to HCQ: mixture of diastereoisomers of 4[(7-chloroquinolin- 4-yl)amino]-N-ethyl-N-(hydroxyethyl)pentan-1-amine N-oxide; 2-[[(4RS)4-[(7-chloroquinolin-4-yl)amino]pentyl]- (ethyl)amino]ethyl hydrogen sulfate; 2-[[(4RS)-4-[(7chloroquinolin-4-yl)amino]pentyl]- amino]ethan-1-ol, D.) D. (4RS)-N4-(7-chloroquinolin-4-yl)N1-ethylpentane-1,4- diamine; (4RS)-4-[(7-chloroquinolin-4-yl)amino]pentan-1-ol; 7-chloro4-[(2RS)-2-methylpyrrolidin-1-yl]quinolone; 4,7-dichloroquinoline. As determined by quantitative NMR, the level of impurities was below the tolerated level described in the EP. Furthermore, NMR confirmed an acceptable level of residual solvents, such as methanol and ethanol $(<0.2 \%(w / w))$.

High resolution electro-spray ionization mass spectrometry (HR-ESI-MS) was performed to corroborate the molecular formula with respect to atoms that are not easily detectable by NMR as e.g. chlorine, nitrogen and oxygen. Pure API and finely powdered tablet both were in agreement with the net formula for the cation of HCQ sulfate. Deviation between theoretical and measured values was less than 2 ppm: (m/z) calculated for $\mathrm{C}_{18} \mathrm{H}_{27} \mathrm{ClN}_{3} \mathrm{O}^{+}$of 336.1837 was in agreement with measured $336.1840[\mathrm{M}+\mathrm{H}]^{+}$(pure API) and $336.1843[\mathrm{M}+\mathrm{H}]^{+}$(tablet) (Figure S4). 
A

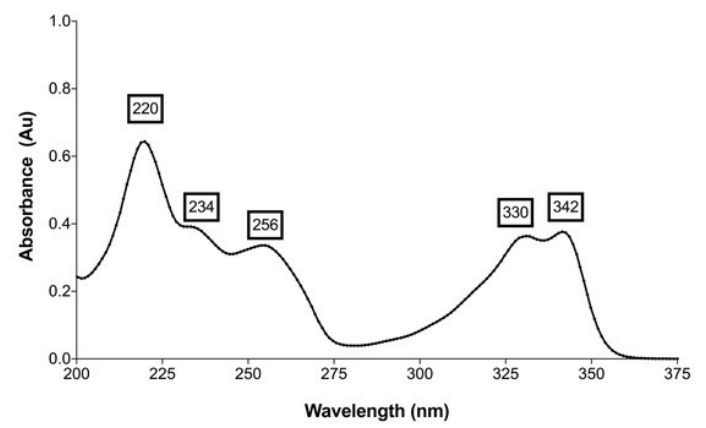

C

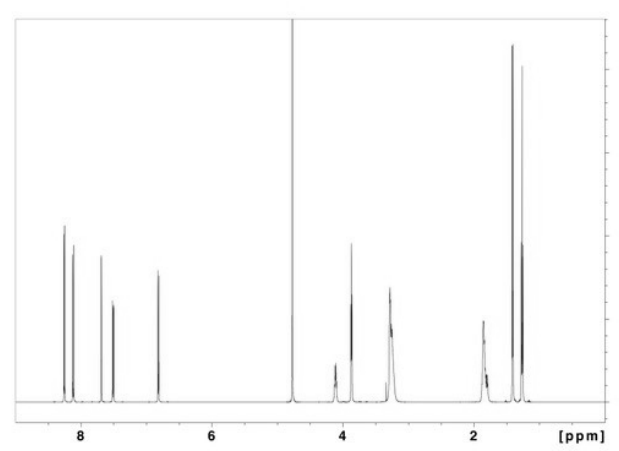

B

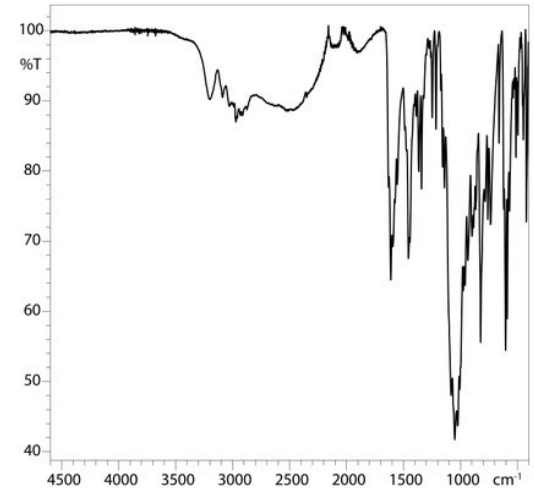

D

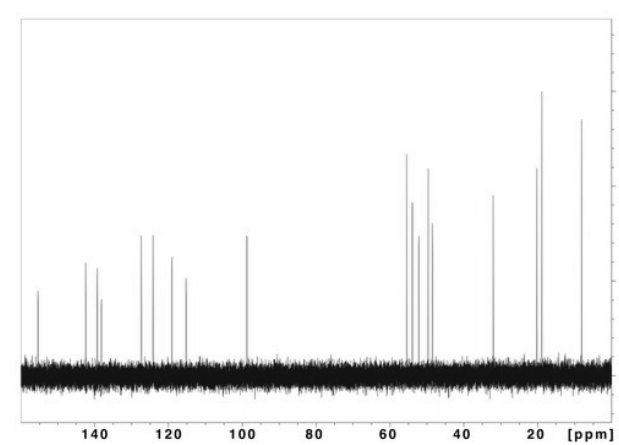

Figure 3. Spectroscopic analysis of HCQ tablets. A) UV Absorption spectrum of HCQ with characteristic absorption peaks B) FT-IR spectrum of Hydroxychloroquine. C) ${ }^{1} H$ NMR (600 $M H z$, in $\left.D_{2} O\right)$ spectrum of HCQ. D $){ }^{13} C N M R\left(151 \mathrm{MHz}\right.$, in $\left.D_{2} O\right)$ spectrum of HCQ. Detailed twodimensional NMR spectra (COSY, NOESY, HSQC, HMBC) are provided as supplementary information.

\section{Preparation of tablets}

The currently available formulations of $\mathrm{HCQ}$ (Plaquenil ${ }^{\circledR}$ ) comes in a film coted immediate release tablet of $155 \mathrm{mg} \mathrm{HCQ}$ base (200 mg salt). According to the specification, excipients used in the formulation include lactose monohydrate, maize starch, magnesium stearate, polyvidone and Opadry OY-L-28900 (containing hypromellose, macrogol 4000, titanium dioxide (E171), lactose). Total parameters of the tablets are $293 \mathrm{mg}$ and approximately $270 \mathrm{~mm}^{3}$. The protective film coating of the tablet is not only protecting the tablet's core but also masks the bitter taste of the API and facilitates swallowing of the tablet. The Plaquenil ${ }^{\circledR}$ 
formulation itself is an immediate release dosage form with greater than $70 \%(w / w)$ drug load. The compatibility and compressibility of such formulations with concentrations of an API greater than the percolation thresholds are governed by the physico-chemical and mechanical properties of the drug substance. The same concept applies equally to powders' behavior during high shear granulation process, which is a critical unit operation for this composition (11).

During wet granulation, the temperature of the powder bulk was gradually increasing. The reason for this is formation of liquid bridges between solid particles, which provide a closer contact between the solid formulation components, therefore increase inter-granular friction. Further transition of liquid bridge geometries towards funicular stage shifts the process to the state of a stable equilibrium between disruption and creation of liquid bridges, and as a consequence, towards formation of a uniform granular composition.

The granulation process was followed by drying of green granules in a tray dryer until granules' residual moisture content reaches $1.5 \%(w / w)$. Dried granulate has formed weak slugs, those were passed through a $1.25 \mathrm{~mm}$ sieve to obtain uniform granules with excellent flowability properties_(6 g/second flow through an orifice according to Eur. Pharm. 9.0, 2.9.16).

The dried and sieved granulate was first mixed with disintegrant, followed by sequential blending with glidant and lubricant powders. Blending with a glidant prior to addition of a lubricant improved flowability and tablet weight uniformity at the cost of some possible increase in hydrophobic properties of the powder mixture (12). The latter was considered as minor factor due to good water solubility of the main formulation component, i.e., the HCQ sulphate.

Final powder blend was compressed into $9 \mathrm{~mm}$ concave tablets with $9 \mathrm{~mm}$ tablet cap radius of curvature. During tableting it was noticed that granules are susceptible to overcompression manifested as minor capping and decrease in tablet hardness. Tablet compaction was carried out in a non-instrumented tableting press, therefore identification of exact desired pressure range remains an open task (Figure 4). 


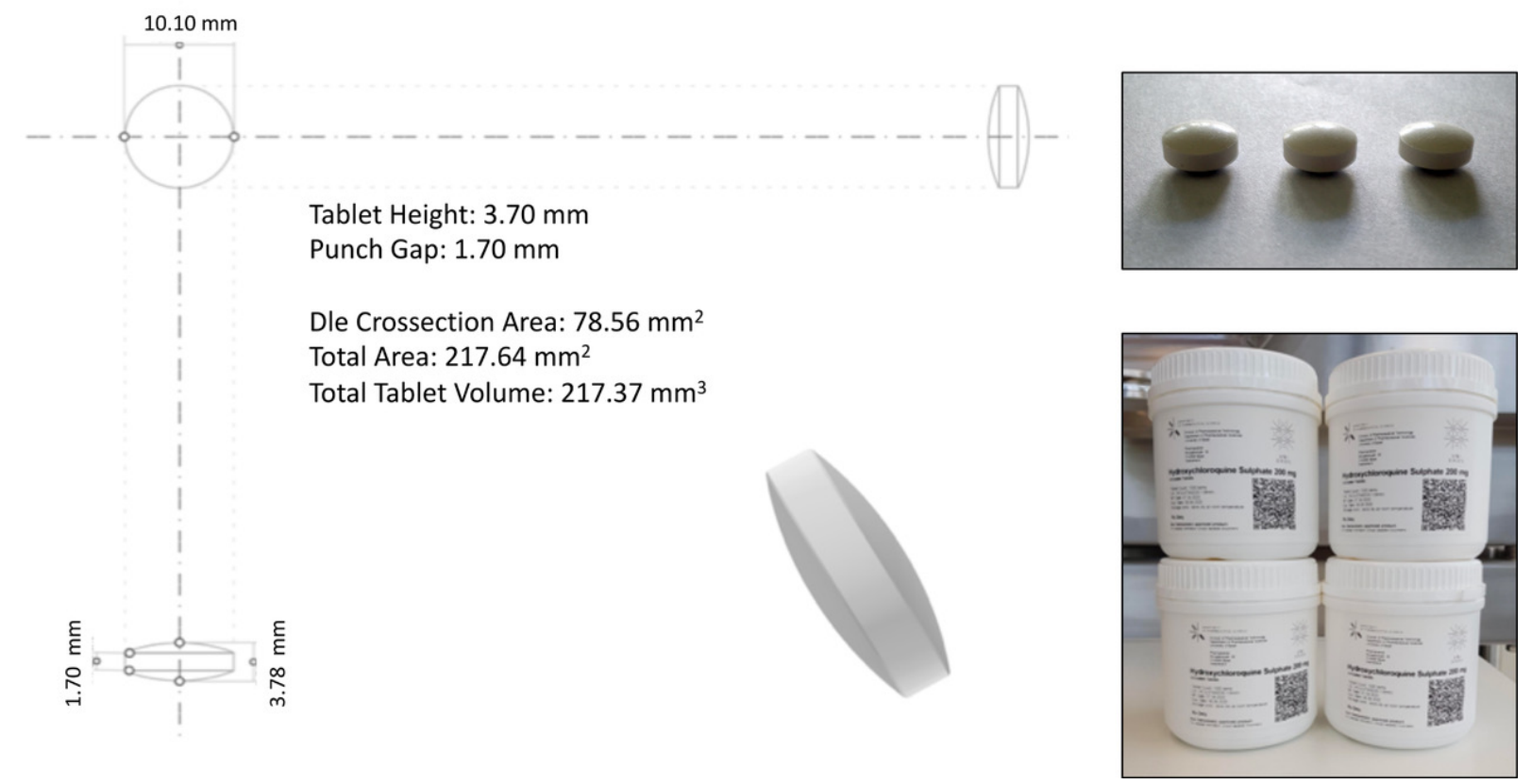

Figure 4. HCQ tablet characteristics, formulation parameters, prototypes of manufactured tablets, and secondary packaging.

\section{Characterization of HCQ tablets}

Manufactured tablets were characterized according to corresponding methods in USP 29 and EP 10. Tablet parameters such as tablet weight, diameter, height, hardness, friability were $299 \pm 2 \mathrm{mg}, 9 \mathrm{~mm}, 3.7 \mathrm{~mm}, 72.8 \pm 11 \mathrm{~N}, 0.1 \%$ (w/w), respectively. The weight and dimensions of our manufactured HCQ tablets are comparable to the commercial HCQ formulation, i.e. Plaquenil ${ }^{\circledR}$.

\section{Drug content and content uniformity}

The measured HCQ dose per tablet showed an average drug content of $98.8 \pm 5 \%$ of the target dose. This is in line with requirements of the Eur. Pharm 9.0, 2.9.6.

\section{Dissolution and disintegration studies}

Dissolution profiles of Plaquenil ${ }^{\circledR}$ and our drug formulation were comparable (Figure 5). Both formulations release approximately $70 \%$ of drug within 30 minutes and more than $90 \%$ within 60 minutes. Requirements set forth in USP 29 are thus fulfilled. Drug release of our tablets is slightly slower as compared to the marketed formulation. However, our tablets release drug 
in a more coherent manner as compared to Plaquienil ${ }^{\circledR}$ tablets. With regard to the results of the disintegration tests, both originator and our uncoated HCQ tablets disintegrated in less than 15 minutes, fulfilling the requirement for immediate release.
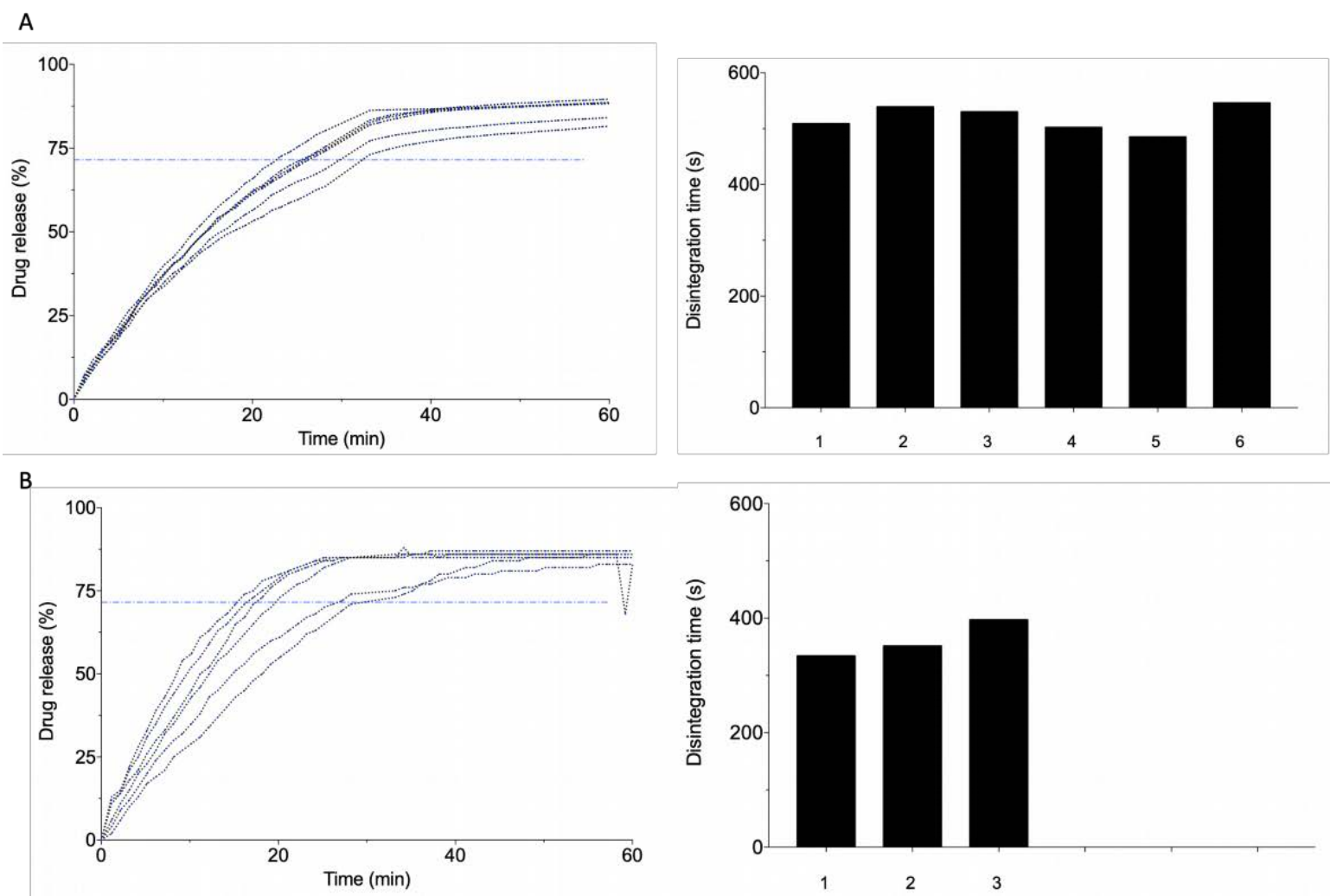

Figure 5. Dissolution and disintegration of HCQ tablets. A) Drug release ( $n=6)$ (left) and disintegration time of $n=6$ HCQ tablets. B) Drug release $(n=6)$ (left) and disintegration time of $n=3$ Plaquenil $^{\circledast}$ tablets. The dotted reference line indicates $70 \%$ of released drug content. 


\section{Conclusion}

The COVID-19 pandemic has exposed the fragility of the global drug manufacturing and distribution system. This situation was of concern to patients suffering from pre-existing diseases, which were confronted with acute drug shortages for HCQ. In addition, developing countries had to face increasing costs for a WHO listed essential medicine against malaria. We therefore provide non-proprietary protocols covering analytical procedures and manufacturing of a HCQ generic. End users are invited to freely use, share, and modify our protocols. Our oral dosage form for HCQ was prepared using a cost-effective formulation strategy, readily available excipients, and a low number of units of operation. This should make our protocols adaptable to small- as well as large-scale manufacturing. Tablets are not coated. This reduces production costs and allows patients with swallowing difficulties to suspend crushed tablets in liquid containing, if necessary, taste masking components (13). In vitro profiling demonstrates close similarity of our generic drug formulation to FDA approved Plaquenil ${ }^{\circledR}$. Considering recent FDA recommendations, it should be possible to apply for a bioequivalence waiver for immediate release formulations.

\section{Acknowledgements}

We are most grateful to Dr. M. Pfeffer and to P. Rieder (University of Basel) for performing the HR-ESI-MS spectrometry. We thank M. Nowak, P. Tschopp, A. Goeth and L. Soeren (Glatt AG, Binzen, Germany) for providing pharmaceutical excipients as a gift. We appreciate the help of Dr. S. Deuster with regulatory questions. 


\section{References}

1. Tanenbaum L, Tuffanelli DL. Antimalarial Agents: Chloroquine, Hydroxychloroquine, and Quinacrine. Arch Dermatol. 1980 May 1;116(5):587-91.

2. Ben-Zvi I, Kivity S, Langevitz P, Shoenfeld Y. Hydroxychloroquine: From Malaria to Autoimmunity. Clin Rev Allergy Immunol. 2012;42(2):145-53.

3. Schrezenmeier E, Dörner T. Mechanisms of action of hydroxychloroquine and chloroquine: implications for rheumatology. Nature Reviews Rheumatology. 2020 Mar;16(3):155-66.

4. Tett SE, Cutler DJ, Day RO, Brown KF. Bioavailability of hydroxychloroquine tablets in healthy volunteers. British Journal of Clinical Pharmacology. 1989;27(6):771-9.

5. Balevic SJ, Green TP, Clowse MEB, Eudy AM, Schanberg LE, Cohen-Wolkowiez M. Pharmacokinetics of Hydroxychloroquine in Pregnancies with Rheumatic Diseases. Clin Pharmacokinet. 2019;58(4):525-33.

6. Lentini G, Cavalluzzi MM, Habtemariam S. COVID-19, Chloroquine Repurposing, and Cardiac Safety Concern: Chirality Might Help. Molecules. 2020 Jan;25(8):1834.

7. Colson P, Rolain J-M, Raoult D. Chloroquine for the 2019 novel coronavirus SARS-CoV2. International Journal of Antimicrobial Agents. 2020 Mar 1;55(3):105923.

8. Self WH, Semler MW, Leither LM, Casey JD, Angus DC, Brower RG, et al. Effect of Hydroxychloroquine on Clinical Status at 14 Days in Hospitalized Patients With COVID19: A Randomized Clinical Trial. JAMA. 2020 Dec 1;324(21):2165-76.

9. FDA-2007-D-0369. Product-Specific Guidances for Chloroquine Phosphate and Hydroxychloroquine Sulfate [Internet]. FDA; 2020 [cited 2020 Apr 29]. Available from: https://www.fda.gov/regulatory-information/search-fda-guidancedocuments/product-specific-guidances-chloroquine-phosphate-andhydroxychloroquine-sulfate

10. Hoffman RE. Standardization of chemical shifts of TMS and solvent signals in NMR solvents. Magn Reson Chem. 2006 May;44(6):606-16.

11. Leuenberger $\mathrm{H}$, Puchkov $\mathrm{M}$, Krausbauer $\mathrm{E}$, Betz G. Manufacturing pharmaceutical granules: Is the granulation end-point a myth? Powder Technology. 2009 Jan 31;189(2):141-8.

12. Pingali K, Mendez R, Lewis D, Michniak-Kohn B, Cuitino A, Muzzio F. Mixing order of glidant and lubricant - Influence on powder and tablet properties. Int J Pharm. 2011 May 16;409(0):269-77.

13. Pauli E, Joshi H, Vasavada A, Brackett J, Towa L. Evaluation of an Immediate-Release Formulation of Hydroxychloroquine Sulfate With an Interwoven Pediatric TasteMasking System. Journal of Pharmaceutical Sciences. 2020 Apr 1;109(4):1493-7. 
Supplementary Figures

A)

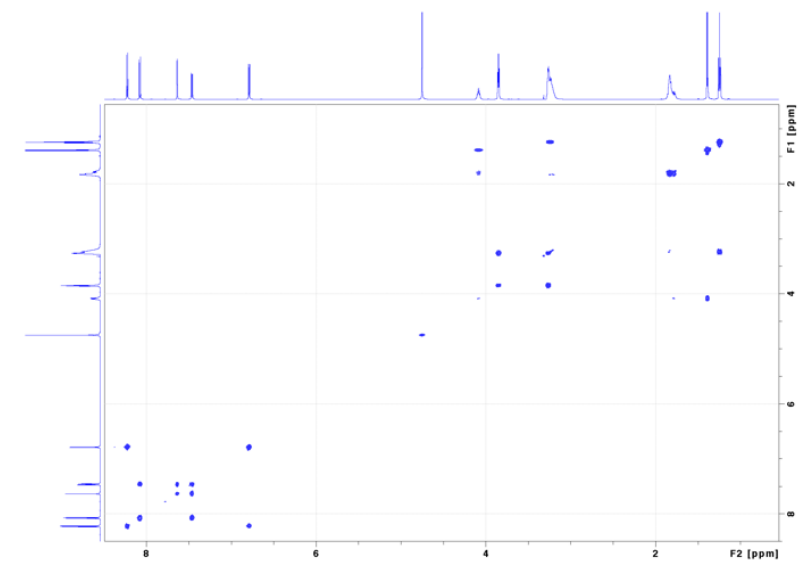

C)

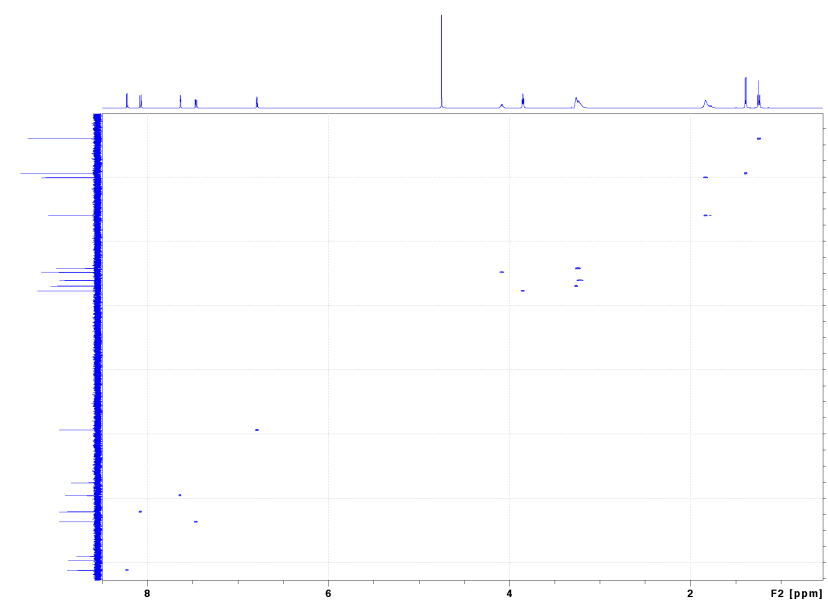

B)

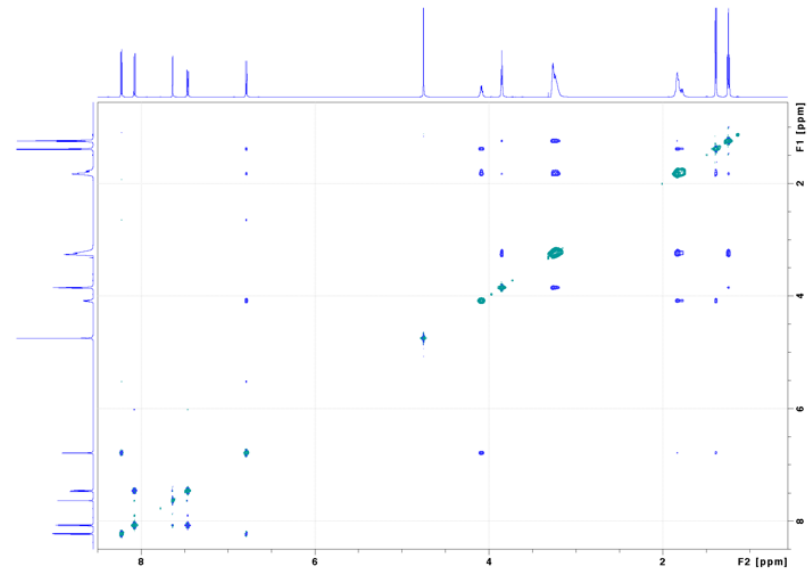

D)

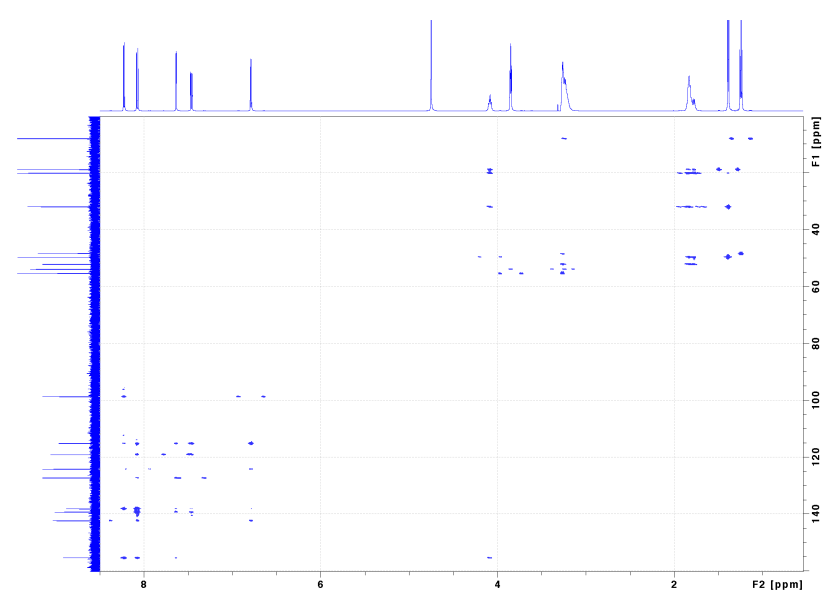

Figure S1. NMR analysis of HCQ. A) Correlated Spectroscopy (COSY). B) Nuclear Overhauser Enhancement Spectroscopy (NOESY). C) Heteronuclear Single Quantum Correlation Spectrum (HSQC). D) Heteronuclear Multiple Bond Spectrum (HMBC). 

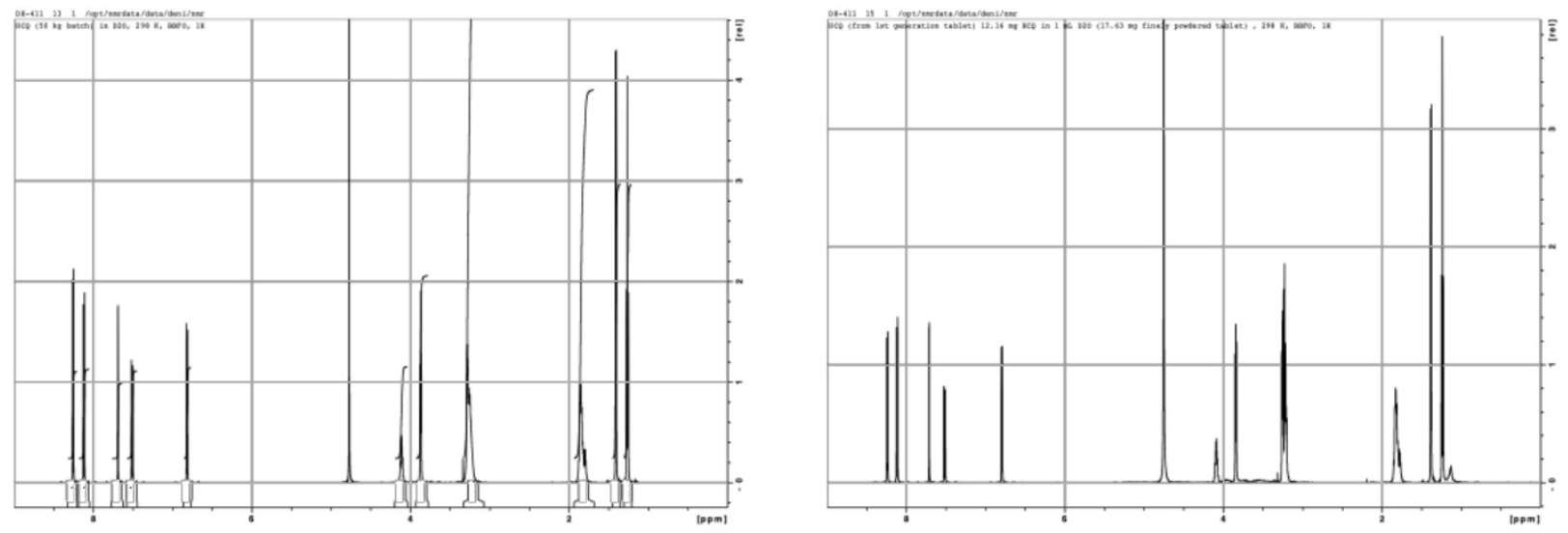

Figure S2. NMR analysis of HCQ. ${ }^{1} H$ NMR spectrum of HCQ before tableting (left panel) and after tableting (right panel).

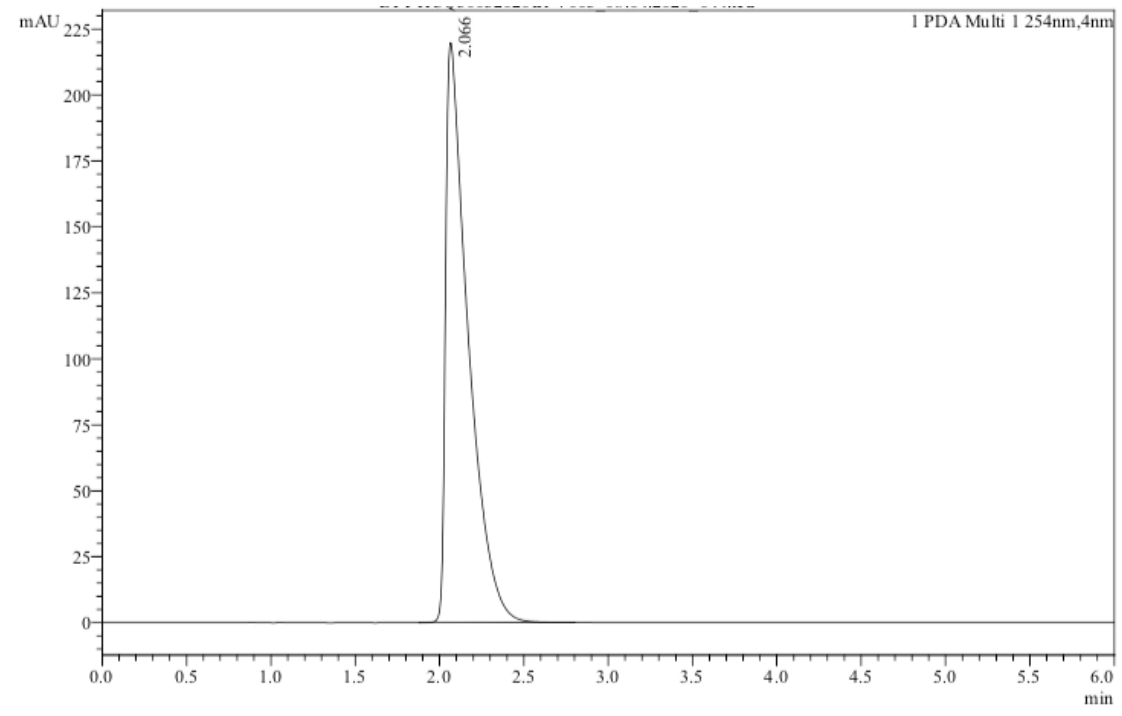

Figure S3. UPLC elution profile of HCQ. 

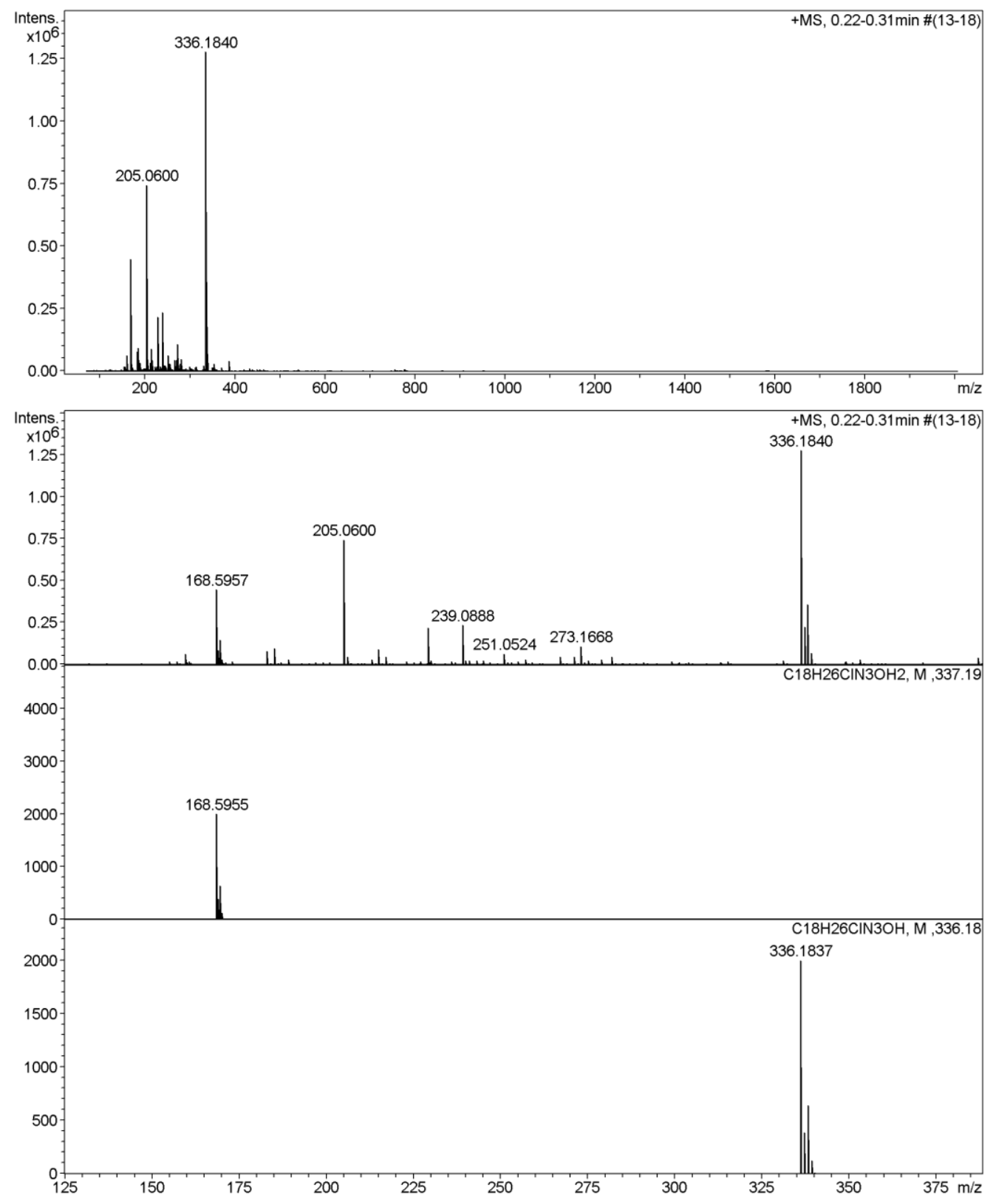

Figure S4. HR-ESI-MS spectra of HCQ. 

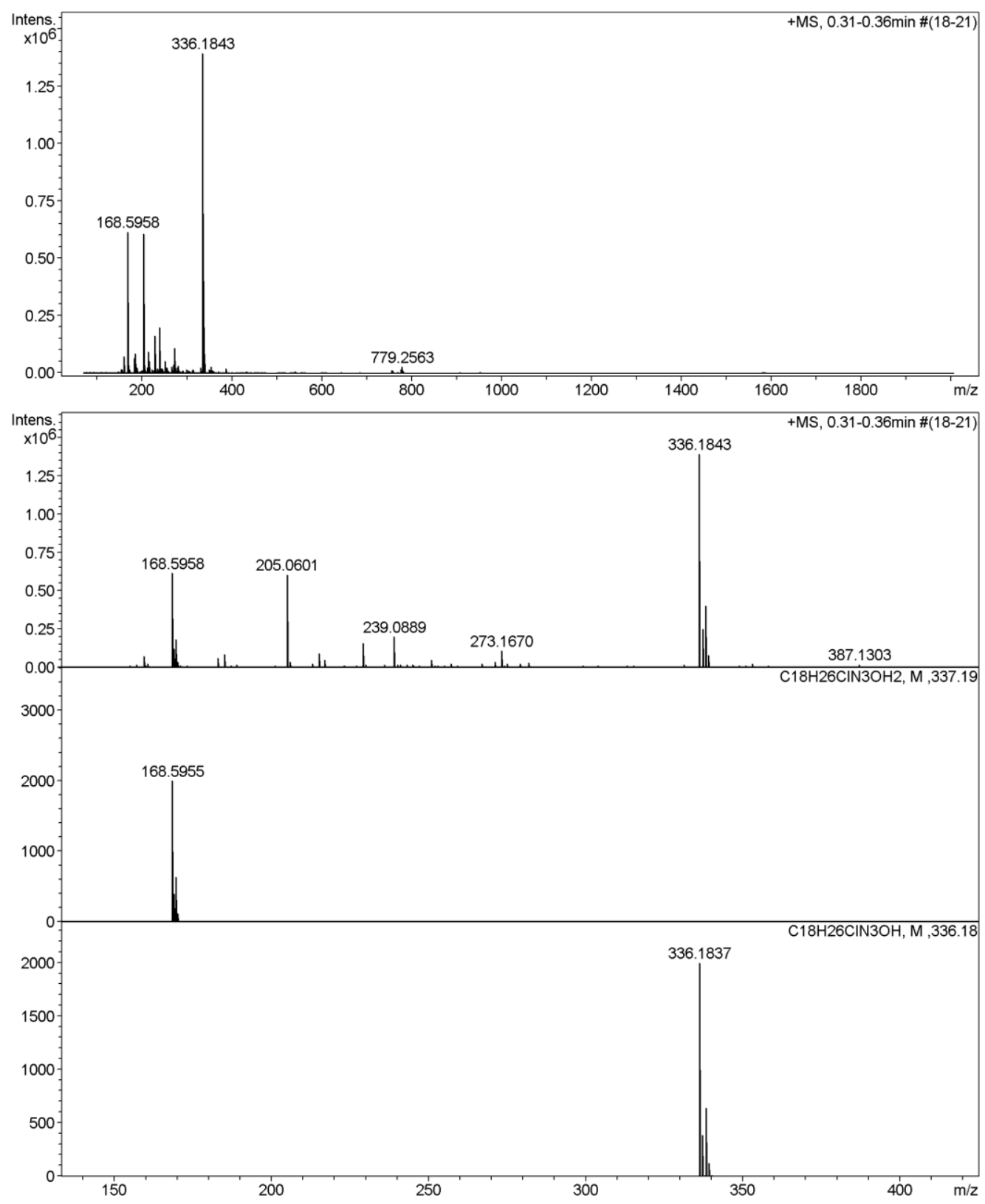

Figure S5. HR-ESI-MS spectra of the HCQ tablet formulation. 